\title{
Frontières
}

\section{La mort au tableau noir}

Mort et deuil dans la classe

\section{Denis Jeffrey}

Volume 13, numéro 1, automne 2000

La mort au tableau noir

URI : https://id.erudit.org/iderudit/1074237ar

DOI : https://doi.org/10.7202/1074237ar

Aller au sommaire du numéro

Éditeur(s)

Université du Québec à Montréal

ISSN

1180-3479 (imprimé)

1916-0976 (numérique)

Découvrir la revue

Citer ce document

Jeffrey, D. (2000). La mort au tableau noir : mort et deuil dans la classe.

Frontières, 13(1), 5-5. https://doi.org/10.7202/1074237ar

Ce document est protégé par la loi sur le droit d'auteur. L'utilisation des services d'Érudit (y compris la reproduction) est assujettie à sa politique d'utilisation que vous pouvez consulter en ligne.

https://apropos.erudit.org/fr/usagers/politique-dutilisation/
Cet article est diffusé et préservé par Érudit.

Érudit est un consortium interuniversitaire sans but lucratif composé de l’Université de Montréal, l'Université Laval et l'Université du Québec à Montréal. Il a pour mission la promotion et la valorisation de la recherche. https://www.erudit.org/fr/ 


\begin{abstract}
Denis Jeffrey,
responsable du thème, professeur au Département d'études sur l'enseignement et l'apprentissage, Faculté des sciences de l'éducation de I'Université Laval.
\end{abstract}

À partir de quel âge peut-on parler de la mort avec les enfants ? Pourquoi devrait-on parler de la mort avec eux ? Vont-ils comprendre ce qu'on leur dit sur la mort? Lorsque survient un décès dans le cadre scolaire, devons-nous en discuter avec les élèves ? Les réponses à ces questions ne sont pas uniques. Or, tous les auteurs de ce numéro de Frontières s'entendent pour soulever le tabou moderne sur la mort. Cela signifie en premier lieu qu'il est toujours préférable de discuter de la mort avec les enfants, lorsque le contexte le permet, plutôt que de les contraindre au silence. Cette idée demeure au cœur des préoccupations de plusieurs auteurs du présent numéro de Frontières.

L'épreuve d'une mort n'est pas facile à vivre pour personne. Les adultes savent bien que la mort est dérangeante, et quelquefois terrifiante. C'est peut-être bien pourquoi nombre d'entre eux aimeraient protéger les enfants contre la connaissance de la mort. Du moins, ils aimeraient conserver le plus longuement possible les enfants dans une sorte d'innocence. Ils ne désirent pas bousculer les enfants également parce qu'ils les croient trop fragiles. Ce mythe de l'innocence infantile a la peau dure. Les enfants, soutiennent Michel Hanus, MarieAnge Abras et Lucie Fréchette dans leur texte, sont capables d'absorber les événements concernant la mort justement si nous leur donnons l'occasion d'en discuter. Les bâillonner dans le silence ne serait d'aucune aide, ni pour l'enfant ni pour l'adulte.

Sidérés par le décès d'un élève de la classe, des enseignants se sentent incapables d'en parler avec les autres élèves. Il n'y a rien d'anormal dans cette réaction. Chacun doit prendre le temps d'amortir le choc des émotions intenses qu'il vit. Dans cette situation, un enseignant peut demander l'aide de la direction, d'un collègue ou d'un professionnel. Josée Masson, dans un entretien avec Pierre-Alexandre Poirier, souligne qu'il est tout à fait normal de laisser une autre personne intervenir à notre place lorsque l'on se croit incapable de le faire.

On sait que les enfants ne conçoivent pas tous la mort de la même manière. Selon l'âge et selon la culture familiale et sociale, l'idée qu'ils se font de la mort, de l'au-delà, du cycle du temps et de la souffrance sera dif- férente. Josef Brynczka, Lucie Fréchette et PierreAlexandre Poirier abordent ce thème, dans leur texte, en le mettant en relation avec les interventions pédagogiques d'un enseignant dans sa classe. Les activités, en classe, pour amorcer une discussion sur la mort sont nombreuses. Charlotte Guérette note que la littérature d'enfance et de jeunesse est riche en thèmes concernant la mort, la peur de la mort, les créatures de la mort et... les joies de la vie. Marie-Ange Abras, pour sa part, rend compte de sa recherche, menée dans le cadre scolaire, qui vise à initier de jeunes élèves au thème de la mort.

Il ne faut certes pas attendre un événement tragique pour parler de la mort avec les enfants. Or, quand survient le décès d'un élève ou d'un membre de l'école, il y a lieu, note Pierre-Alexandre Poirier, de ritualiser le deuil. Yves Marcoux suggère de prendre en considération les relations d'affection vécues entre les jeunes lorsque survient le décès de l'un d'eux. Pour ce qui est de la direction scolaire, Sylvie Donnadieu et Pascale Moreau proposent un protocole pour gérer une situation de mortalité. On sait l'importance de ces protocoles lorsque survient notamment un suicide. Dans son article, Robert Verreault examine comment les médias ont présenté la fusillade dans une école secondaire de Littleton. Il découvre que la rumeur et le mythe ont complètement faussé la réalité des événements.

Dans des textes plus personnels, Guy Bourgeault et Denis Jeffrey présentent leurs réflexions sur la mort. Chacun évoque des souvenirs, des lectures et des moments philosophiques qui ont enrichi et intensifié leur méditation. En plus des articles présentant un caractère scientifique et philosophique, on retrouve dans ce numéro deux textes littéraires. Le premier, écrit par Denise Blais, met en scène la tristesse et le désarroi d'une fillette qui vient tout juste de perdre une amie. Le second, sous la plume de Fernande Goulet, utilise le style épistolaire pour donner la parole à une grand-mère qui parle à sa petite-fille du temps qui passe, de la vieillesse et de la mort. Afin de donner la parole aux enfants, nous avons demandé à Luce Des Aulniers de rendre compte de deux films documentaires qui abordent le thème de la mort à l'enfance.

En terminant ce numéro, je tiens à remercier l'équipe de Frontières ainsi que mes amis Clemon George et Bernard Jobin pour leur aide dans la traduction, dans la correction et dans la recherche bibliographique. 